\title{
Metabolic syndrome in women - correlation between BMI and waist circumference
}

\author{
Marcin Gierach ${ }^{\circledR}$, Roman Junik ${ }^{\circledR}$ \\ Department of Endocrinology and Diabetology, Collegium Medicum in Bydgoszcz, Nicolaus Copernicus University in Toruń, \\ Bydgoszcz, Poland
}

\begin{abstract}
Introduction: Metabolic syndrome (MetS), which includes abdominal obesity, hypertension, hypertriglyceridaemia, low high-density lipoprotein cholesterol and elevated fasting plasma glucose, is now well understood. The prevalence of MetS is increasing all over the world. Central obesity is the main and the most common component of MetS. The most simple, practical, and frequently used tool for assessing body weight is body mass index. The aim of our study was to show the correlation between waist circumference (WC) and BMI in women with MetS depending on its components.

Material and methods: It was a study of 36-month duration (2016-2019) conducted in University Hospital No. 1 in Bydgoszcz, Poland. The study included 696 women with diagnosed MetS according to the 2009 International Diabetes Federation criteria.

Results: In the study group, WC was found to be significantly correlated with BMI according to the arterial hypertension, T2DM, IFG, lower HDL-C, and higher TG level.

Conclusion: We should consider changing the WC guidelines for Europeans, but for this we need more prospective studies. (Endokrynol Pol 2022; 73 (1): 163-164)

Key words: metabolic syndrome; waist circumference; BMI
\end{abstract}

\section{Introduction}

Metabolic syndrome (MetS), which includes abdominal obesity, arterial hypertension (AH), hypertriglyceridaemia ( $\uparrow$ TG), low HDL-C, and elevated fasting plasma glucose, is now well understood [1]. The prevalence of MetS is increasing all over the world. It is worth noting the observation that the increase of MetS is greater in women than in men [2]. What is important, the prevalence of MetS increases with age [1]. The most simple, practical, and frequently used tool for assessing body weight is body mass index (BMI), but we must be aware of its limitations. BMI ignores the physiological differences in the proportions of the tissues such as adipose, osseous, and muscular. The aim of our study was to show the correlation between waist circumference (WC) and BMI in women with MetS depending on its components.

\section{Material and methods}

It was a study of 36-month duration (2016-2019) conducted in University Hospital No. 1 in Bydgoszcz, Poland. The study included 696 women with diagnosed MetS according to the 2009 International Diabetes Federation (IDF) criteria. BMI was calculated as body weight (in kilograms) divided by the square of body height (in metres). WC was measured halfway between the lowest rib and the top of the hipbone. We followed the methods of Gierach et al. 2014 [3].

\section{Results}

In the study group, WC was found to be significantly correlated with BMI according to $\mathrm{AH}$, type 2 dabetes mellitus (T2DM), IFG, $\downarrow$ HDL-C, and $\uparrow$ TG (Tab. 2).

\section{Discussion}

The constantly increasing population of patients diagnosed with MetS is a leading health concern all over the world [4]. Janszky et al. [5] said that the prevalence of MetS is greater in women than in men (49.9\% vs. 34.3\%), and it increases with age. Also, in Poland MetS is a very serious social problem that is growing. The NATPOL 2002 study shows MetS in 19.7\% of women, and the average WC in women is $86.5 \mathrm{~cm}$. In the subgroups 18-39 years, $40-59$ years, and $60-79$ years, the results were $79.3 \mathrm{~cm}, 87.6 \mathrm{~cm}$, and $95.7 \mathrm{~cm}$, respectively, in females [6]. After almost 10 years as much as $26 \%$ of the Polish population were diagnosed with MetS, as was shown in the 2011 NATPOL PLUS study. Abdominal obesity, which is the most common component of MetS, occurs in about 6.5 million people in Poland. It is predicted that this number will increase to as much as 10 million during the next 10-15 years. The second most frequent component was $\mathrm{AH}(71 \%)$, followed by glucose metabolism disturbances (37.3\%), $\uparrow$ TG (21.2\%), and $\downarrow$ HDL 
Table 1. General characteristics of the study group

\begin{tabular}{lccccccc}
\hline Women & $\mathbf{N}(\%)$ & Age [years] & SD & BMI [kg/m2] & SD & WC [cm] & SD \\
\hline Total & $696(100)$ & 67.5 & 11.9 & 30.7 & 5.5 & 105.9 & 22.5 \\
\hline Abdominal obesity & $678(97.4)$ & 67.4 & 12.0 & 30.9 & 5.4 & 108.2 & 21.7 \\
\hline AH & $650(93.4)$ & 67.9 & 12.4 & 30.8 & 5.7 & 108.0 & 19.7 \\
\hline IFG & $140(20.2)$ & 63.6 & 14.2 & 30.3 & 5.2 & 102.6 & 27.7 \\
\hline T2DM & $412(59.2)$ & 68.4 & 11.1 & 31.2 & 5.9 & 109.3 & 23.9 \\
\hline$\uparrow$ TG & $256(36.8)$ & 63.8 & 11.7 & 30.4 & 4.9 & 103.6 & 23.1 \\
\hline$\downarrow$ HDL-C & $392(56.3)$ & 68.1 & 11.1 & 30.9 & 5.3 & 108.1 & 18.9 \\
\hline
\end{tabular}

AH — arterial hypertension; IFG — impaired fasting glucose; T2DM — type 2 diabetes mellitus; TG — triglycerides; HDL-C — high-density lipoprotein cholesterol

Table 2. General correlation between body mass index (BMI) and waist circumstance (WC) in the study group, according to arterial hypertension (AH), type 2 diabetes mellitus (T2DM), impaired fasting glucose (IFG), decreased high-density lipoprotein cholesterol ( $\downarrow H D L-C)$, and increased triglyceride ( $\uparrow T G)$

\begin{tabular}{|c|c|c|c|c|c|c|}
\hline $\mathrm{BMI} / \mathrm{WC}$ & \multicolumn{3}{|c|}{$\mathrm{AH}$} & \multicolumn{3}{|c|}{ Arterial normotension } \\
\hline Women & $\mathrm{n}=650$ & $r=0.81$ & $p<0.05$ & $\mathrm{n}=46$ & $r=0.65$ & $p<0.05$ \\
\hline BMI/WC & \multicolumn{3}{|c|}{ T2DM } & \multicolumn{3}{|c|}{ No carbohydrate disorders } \\
\hline Women & $\mathrm{n}=412$ & $r=0.83$ & $p<0.05$ & $\mathrm{n}=144$ & $r=0.79$ & $p<0.05$ \\
\hline BMI/WC & \multicolumn{3}{|c|}{ IFG } & \multicolumn{3}{|c|}{ No carbohydrate disorders } \\
\hline Women & $n=140$ & $r=0.78$ & $p<0.05$ & $\mathrm{n}=144$ & $r=0.81$ & $p<0.05$ \\
\hline BMI/WC & \multicolumn{3}{|c|}{ Decreased HDL-C } & \multicolumn{3}{|c|}{ Normal HDL-C } \\
\hline Women & $\mathrm{n}=392$ & $r=0.80$ & $p<0.05$ & $\mathrm{n}=304$ & $r=0.83$ & $p<0.05$ \\
\hline BMI/WC & \multicolumn{3}{|c|}{ Increased TG } & \multicolumn{3}{|c|}{ Normal TG } \\
\hline Women & $n=151$ & $r=0.76$ & $p<0.05$ & $\mathrm{n}=304$ & $r=0.83$ & $p<0.05$ \\
\hline
\end{tabular}

$(15.9 \%)$ [5]. In our study, we observed that correlation occurred between BMI and WC in each of the studied subgroups. In the subgroup of $\mathrm{AH}$, the correlation coefficient was the lowest $(\mathrm{r}=0.65)$. This was probably due to the small size of the subgroup with normal arterial blood pressure $(n=46)$ and lower $B M I$ and $W C$ values vs. the subgroup with AH (BMI 28.6 vs. 30.8, respectively, and WC 104.3 vs. 108.0, respectively). The results obtained in the group with BMI $=30 \mathrm{~kg} / \mathrm{m}^{2}$ correlate with a WC of $102 \mathrm{~cm}$ in women. In the European female population abdominal obesity is currently defined as $W C \geq 80 \mathrm{~cm}$, which corresponds to $\mathrm{BMI}=21.62 \mathrm{~kg} / \mathrm{m}^{2}$. The question is whether the obesity criteria currently in force, based on BMI calculation, should be reviewed or if the usage of these criteria to assess abdominal body fat excess should be abandoned. On the other hand, WC analysis can be burdened with some measurement limitations resulting from the different measurement sites, e.g. umbilicus, hip crest, and minimal waist, which can lead to diagnostic problems. However, the difference in WC measurement is reduced by the sample size and the high occurrence of obesity among patients [7]. Therefore, WC seems to be a preferable predictor of the risk factors of MetS development compared to BMI [8].

We believed that there is a strong linear correlation between WC and BMI values in women with MetS re- gardless of its components. The presence of even normal body weight in females (BMI $21.62 \mathrm{~kg} / \mathrm{m}^{2}$ ) corresponds to an increased amount of abdominal adipose tissue, so we should consider changing the WC guidelines for Europeans, but for this we need more prospective studies.

\section{References}

1. Balkau B. Epidemiology of the metabolic syndrome and the RISC study. Eur Heart J Suppl. 2005; 7(suppl_D): D6-D9, doi: 10.1093/eurheartj/sui022.

2. Mozumdar A, Liguori G. Persistent increase of prevalence of metabolic syndrome among U.S. adults: NHANES III to NHANES 1999-2006. Diabetes Care. 2011; 34(1): 216-219, doi: 10.2337/dc10-0879, indexed in Pubmed: 20889854

3. Gierach M, Gierach J, Ewertowska M, et al. Correlation between Body Mass Index and Waist Circumference in Patients with Metabolic Syndrome. ISRN Endocrinol. 2014; 2014: 514589, doi: 10.1155/2014/514589, indexed in Pubmed: 24729884.

4. Janghorbani M, Amini M, Janghorbani M, et al. Incidence of and risk factors for diabetic retinopathy in Isfahan, Iran. Ophthalmic Epidemiol. 2003; 10(2): 81-95, doi: 10.1076/opep.10.2.81.13893, indexed in Pubmed: 12660857.

5. Janszky I, Vatten L, Romundstad P, et al. Metabolic syndrome in Poland - the PONS Study. Ann Agric Environ Med. 2011; 18(2): 270-272, indexed in Pubmed: 22216795.

6. Zdrojewski T, Rutkowski M, Bandosz T, et al. Prevalence and control of cardiovascular risk factors in Poland. Assumptions and objectives of the NATPOL 2011 Survey. Kardiol Pol . 2013; 71(4): 381-392, doi: 10.5603/KP.2013.0066.

7. Sumner AE, Micklesfield LK, Ricks M, et al. Waist circumference, BMI, and visceral adipose tissue in white women and women of African descent. Obesity (Silver Spring). 2011; 19(3): 671-674, doi: 10.1038/oby.2010.201, indexed in Pubmed: 20847732

8. Aye M, Sazali M. Waist circumference and BMI cut-off points to predict risk fctors for metabolic syndrome among outpatients in a district hospital. Singapure Med J. 2012; 53(8): 545-550, indexed in Pubmed: 22941134. 